APPENDICE 1

\title{
Quelques notions de base en biogéochimie
}



Il s'agit de notions classiques permettant de décrire l'état et la dynamique du fonctionnement d'un écosystème complexe; celles-ci sont utilisées notamment lors de mise au point de modèles (cf. chapitre 9).

Les compartiments représentent des entités finies supposées de composition et de comportement homogène à une échelle fixée (aucune distribution n'est considérée dans un compartiment). Leur limite spatiale est définie et leur évolution temporelle peut être abordée par des mesures successives. Dans un écosystème forestier par exemple, les principaux compartiments sont la végétation et les sols. Ces derniers sont divisés en sous-compartiments qui permettent de préciser la description de l'objet étudié. Ainsi, pour la végétation chaque étage peut être séparé en partie pérenne et en partie non pérenne (feuilles par exemple). Pour le sol, on identifie en général des sous-compartiments solides organiques ou minéraux et des solutions libres ou liées.

Ces compartiments sont pour l'échelle écosystémique des réservoirs, mais ce vocabulaire n'est que peu employé pour les écosystèmes terrestres, car il est réservé en géochimie à des entités correspondant aux grands types de roches de l'écorce terrestre ou aux océans par exemple. Les échelles temporaires et spatiales n'ont rien à voir avec les écosystèmes dont il est question ici.

Ces compartiments échangent, entre eux et avec l'environnement, de la matière et de l'énergie par des flux de nature solide (exemple : transfert de particules lors de l'érosion), liquide (exemple : transfert d'éléments en solution provenant de l'atmosphère, transférés dans les sols puis vers les eaux de surface ou les nappes profondes), gazeux (transfert de $\mathrm{CO}_{2}$ issu de la respiration des organismes ou de la minéralisation des matières organiques des sols) et enfin d'énergie.

L'intérêt de ces notions, dans le cadre de l'étude des biogéocycles et de leur modélisation, est de permettre la description (pour un élément donné) de la variation d'un compartiment par la somme algébrique des flux entrant et sortant de ce compartiment, pendant une durée fixée. Tous les flux ne sont pas mesurables, mais le système d'équations ainsi défini et le recours à certaines hypothèses simplificatrices, permettent d'estimer par le calcul certains flux inaccessibles à la mesure.

Le temps de résidence est le temps qu'un élément passe en moyenne dans un compartiment. Il dépend de la durée de renouvellement du compartiment et de la réactivité de l'élément dans l'environnement. Après un temps équivalent au temps de résidence, les entrées et les sorties d'un compartiment s'équilibrent. 
